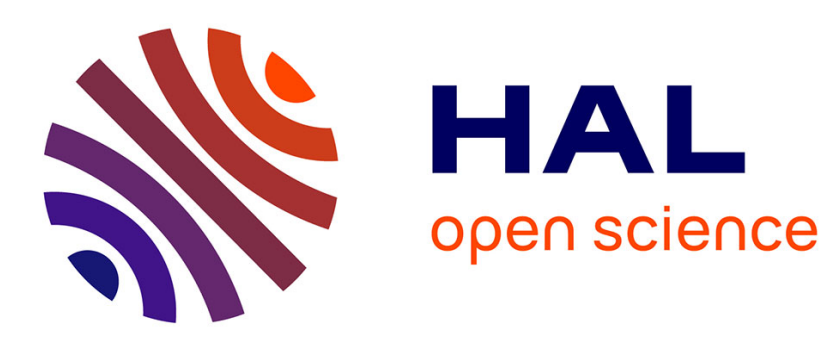

\title{
Photonic crystal waveguides: out-of-plane losses and adiabatic modal conversion
}

Mirel Palamaru, Philippe Lalanne

\section{To cite this version:}

Mirel Palamaru, Philippe Lalanne. Photonic crystal waveguides: out-of-plane losses and adiabatic modal conversion. Applied Physics Letters, 2001, 78 (11), pp.1466-1468. hal-00859065

\section{HAL Id: hal-00859065 \\ https://hal-iogs.archives-ouvertes.fr/hal-00859065}

Submitted on 6 Sep 2013

HAL is a multi-disciplinary open access archive for the deposit and dissemination of scientific research documents, whether they are published or not. The documents may come from teaching and research institutions in France or abroad, or from public or private research centers.
L'archive ouverte pluridisciplinaire HAL, est destinée au dépôt et à la diffusion de documents scientifiques de niveau recherche, publiés ou non, émanant des établissements d'enseignement et de recherche français ou étrangers, des laboratoires publics ou privés. 


\title{
Photonic crystal waveguides: Out-of-plane losses and adiabatic modal conversion
}

\author{
M. Palamaru and Ph. Lalanne ${ }^{\text {a) }}$ \\ Laboratoire Charles Fabry de l'Institut d'Optique, Centre National de la Recherche Scientifique, BP 147, \\ 91403 Orsay Cedex, France
}

(Received 20 October 2000; accepted for publication 15 January 2001)

\begin{abstract}
An accurate model for the out-of-plane radiation losses occurring when a guided wave propagating in a conventional waveguide impinges on a photonic crystal waveguide is presented. The model makes clear that the losses originate from insertion losses resulting from a mode mismatch. A generic taper structure realizing an adiabatic modal conversion is proposed and validated through numerical computations for cavities with large $Q$ 's and large peak transmission. (C) 2001 American Institute of Physics. [DOI: 10.1063/1.1354666]
\end{abstract}

Photonic crystals (PC) etched through waveguides have inspired great interest recently because of their potential use in integrated circuits. These structures can find many applications, including the fabrication of two-dimensional dielectric mirrors ${ }^{1}$ and narrow-band filters, ${ }^{2}$ of ultrashort in-plane microlasers $^{3,4}$ and of waveguides with sharp bends. ${ }^{5,6}$ In PC waveguides, we are resorting to standard index guiding in the vertical direction and light can couple to radiative modes. The reduction of the out-of-plane radiation losses is essential for future applications.

To understand the origin of these losses, we consider the basic scattering problem shown in Fig. 1, where a guided wave propagating in the positive $z$ direction in a conventional waveguide impinges on a PC waveguide. We assume that the wave frequency is in the gap and that the PC waveguide is long enough so that no light is transmitted, the light being reflected or scattered. We also assume that the PC waveguide supports a genuine nonleaky guided mode. ${ }^{7}$ Many experimental observations have reported losses for the considered problem, for planar waveguides perforated with deep grooves ${ }^{3,4}$ or with two-dimensional (2D) arrays of holes, ${ }^{1,8}$ and for ridge waveguides ${ }^{2}$ with one-dimensional (1D) arrays of holes. Although the losses have been previously studied with three-dimensional (3D) electromagnetic analysis $^{9-13}$ or with ingenious perturbation models, ${ }^{8}$ their origin is not clearly understood. It is often admitted that the losses are weaker for strongly confined waveguides, for sub$\lambda / 4$ air-hole widths and for deep etches, the smaller loss (usually called "intrinsic'" losses) being achieved for etches that fully overlap the mode profile of the unperturbed waveguide. In this letter, we propose a model for the losses and show that the losses simply originate from a mode mismatch (provided that a genuine nonleaky guided mode exists). From this understanding, we propose a generic taper structure for adiabatic coupling between conventional waveguides and PC waveguides and predict performances better than the intrinsic ones.

Like the coupled-mode theory ${ }^{14}$ used in integrated optics for the analysis of waveguides with small periodic corrugations, our model assumes that the field in the $\mathrm{PC}$ waveguide

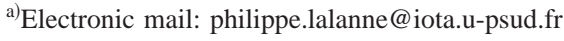

is a linear combination of two contradirectional propagating PC modes. What is meant by PC mode will be precisely defined hereafter. Qualitatively, the losses are seen to be resulting from a triple scattering process in which the incident wave is first scattered into the PC mode propagating into the positive $z$ direction with efficiency $\eta$, then this PC mode is coupled back into the counter-propagating PC mode without loss, ${ }^{7}$ and finally this counter-propagating PC mode is scattered back into the conventional waveguide with efficiency $\eta$ (reciprocity theorem). The out-of-plane losses are simply 1 $-\eta^{2}$. More quantitatively, let us denote by $\mathbf{E}_{1}^{(p)}, p=1,2 \ldots$, the infinite set of the normal modes (propagation constants $\lambda_{p}$ 's) of the conventional waveguide. We also introduce the Bloch modes ${ }^{15} \mathbf{E}_{F}^{(n)}$ of the PC waveguide. Owing to the completeness of the $\mathbf{E}_{1}^{(p)}$ 's, we have

$$
\mathbf{E}_{F}^{(n)}=\sum_{p}\left[a_{p, n}^{+} \exp \left(\lambda_{p} z\right)+a_{p, n}^{-} \exp \left(-\lambda_{p} z\right)\right] \mathbf{E}_{1}^{(p)}(x, y) .
$$

We can now define the PC modes $\mathbf{E}_{T}^{(n)}$ introduced earlier in the qualitative model description as

$$
\mathbf{E}_{T}^{(n)}=\sum_{p} a_{p, n}^{+} \exp \left(\lambda_{p} z\right) \quad \mathbf{E}_{1}^{(p)}(x, y)
$$

for modes propagating in the positive $z$ direction. PC modes propagating in the negative $z$ direction are defined similarly. Let us denote by $\mathbf{E}_{T}^{(1)}$ the nonleaky guided mode supported by the PC waveguide. By use of the classical approximate expression $^{14}$ for the coupling efficiency at an interface between two slightly-different conventional waveguides, we have



FIG. 1. Basic scattering problem considered for modeling out-of-plane the losses when a guided wave impinges on a PC waveguides. The PC waveguide can be a very short $1 \mathrm{D}$ Bragg reflector, a $2 \mathrm{D}$ photonic band gap structure, a strip waveguide with a 1D array of holes, or an even more complex PC structure like that of Fig. 3. 


$$
\eta=\frac{\operatorname{Re}\left\{\left[\iint d x d y\left(\mathbf{E}_{1}^{(1)} \times \mathbf{H}_{T}^{(1) *}\right) e_{z} \iint d x d y\left(\mathbf{E}_{T}^{(1)} \times \mathbf{H}_{1}^{(1) *}\right) \mathbf{e}_{z}\right] /\left[\iint d x d y\left(\mathbf{E}_{T}^{(1)} \times \mathbf{H}_{T}^{(1) *}\right) \mathbf{e}_{z}\right]\right\}}{\operatorname{Re}\left\{\iint d x d y\left(\mathbf{E}_{1}^{(1)} \times \mathbf{H}_{1}^{(1) *}\right) \mathbf{e}_{z}\right\}},
$$

for the coupling efficiency $\eta$ between $\mathbf{E}_{1}^{(1)}$ and $\mathbf{E}_{T}^{(1)}$. In Eq. (3), $\mathbf{e}_{z}$ is the unit vector along the $z$ direction and $\mathbf{H}$ is the standard notation for the magnetic field. The overlap integral of Eq. (3) is all the more accurate as the modes are similar. This amounts to considering that the etches are not deep and large at the same time, the relative amount of removed material in the holes having to remain small.

The model is now tested for PC waveguides composed of 1D periodic structures deeply etched into planar waveguides, ${ }^{3,4}$ an extension of classical Bragg reflectors to strong corrugations, by comparing the out-of-plane losses computed with exact electromagnetic theory ${ }^{16}$ to $1-\eta^{2}$. The $\mathbf{E}_{1}^{(1)}$ and $\mathbf{E}_{T}^{(1)}$ modes required for the overlap integral computation are obtained following Ref. 16. The losses are plotted in Fig. 2 for several etch depths $d$ and polarizations. In Fig. 2(a), a silicon-on-insulator (SOI) waveguide corresponding to a "strong-confinement" case is considered for transverse electric (TE) polarization. The waveguide is composed of a silicon (refractive index 3.505) $205 \mathrm{~nm}$ thick core with claddings with refractive indices 1 and 1.5. In Fig. 2(b), a "weak-confinement" case is considered for TE and transverse magnetic (TM) polarizations. The waveguide is composed of a GaAs (refractive index 3.5) $250 \mathrm{~nm}$ large core layer deposited on a substrate with refractive index 3 . The refractive index of the $400 \mathrm{~nm}$ thick cover is 3 . Within the frequency range of interest i.e., in the band gaps indicated by thin horizontal lines, we find a very good agreement between the losses predicted by the present model (markers) and those provided by exact electromagnetic theory (solid
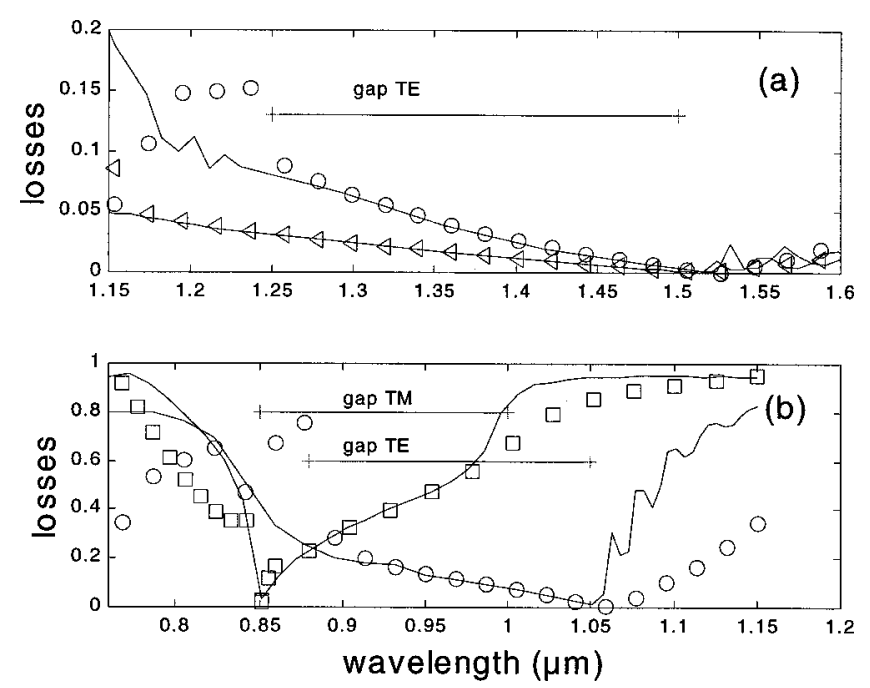

FIG. 2. Comparison between the model predictions (markers) for the losses and exact electromagnetic numerical results (solid curves). (a) First-order Bragg SOI reflector for TE polarization with $L=80, \Lambda=275, d=160$, (circles) and $d=205 \mathrm{~nm}$ (triangles). (b) Second-order Bragg GaAs waveguide with $L=80, \Lambda=330 \mathrm{~nm}$. Squares (resp. circles) are obtained for TM (resp. TE) polarization and for $d=900 \mathrm{~nm}$ (resp. $700 \mathrm{~nm}$ ). The band gap intervals are indicated by horizontal lines. curves). The maximum deviation does not exceed 0.05. Outside the gap, the model is not accurate. It is mainly due to the fact that the PC mode is not coupled back into the counterpropagating PC mode and that it rather propagates backward and forward between the two reflector boundaries in a same way as multiple interference occurs in a thin film. The quantitative agreement in Fig. 2 in the gaps makes us confident on the model validity. It is reasonable to infer that the losses observed through electromagnetic computations ${ }^{9-11}$ or through experiments with $1 \mathrm{D}$-periodic ${ }^{2-4}$ or $2 \mathrm{D}$-periodic ${ }^{8} \mathrm{PC}$ waveguides are mainly due to insertion losses.

Within the scope of the model, we can qualitatively understand the influence of the hole depth $d$ on the amount of losses. For small $d$ values, as in DFB Bragg reflectors for example, $\mathbf{E}_{1}^{(1)}$ and $\mathbf{E}_{T}^{(1)}$ have similar profiles and insertion losses can be neglected, as is usually done for the perturbation approach used in the coupled-mode theory. As $d$ increases, the $\mathbf{E}_{1}^{(1)}$ and $\mathbf{E}_{T}^{(1)}$ profiles start to depart from each other and the overlap integral $\eta$ decreases. As shown by numerical evidence especially for strongly confined waveguides, this decrease is only weakly due to a profile distortion, the prime effect being due to a transverse displacement of $\mathbf{E}_{T}^{(1)}$ relatively to $\mathbf{E}_{1}^{(1)}$. Referring to Fig. 1, the displacement originates from the fact that, in comparison with the unpatterned region of the waveguide, the field experiences a material with a lower refractive index in the top etched region of the waveguide; thus as $d$ increases, the $\mathbf{E}_{T}^{(1)}$ mode is shifted down to the substrate, remaining confined in the unpatterned waveguide region. As $d$ increases further, the transverse displacement still increases, passes through a maximum value, then diminishes to reach an asymptotic weak value $s$ for deep holes fully overlapping $\mathbf{E}_{1}^{(1)}$ (intrinsic case). For symmetric waveguides deeply etched into the substrate or for fully etched membranes in air, $s$ is even null ${ }^{17}$ and losses result only from the mode profile mismatch. The fact that small $s$ values are achieved for deep etches into both weakly- and strongly-confined waveguides is responsible for the fact that short mirrors (or cavities) with acceptable losses are manufacturable with almost any waveguide index profile.

The previous analysis shows the necessity of having an adiabatic impedance matching between conventional and PC waveguides. The design and manufacture of these tapers are almost untreated ${ }^{18}$ in the literature. A generic approach for these tapers is sketched in Fig. 3. The taper is formed by a series of etches whose feature dimensions vary progressively to convert the waveguide mode into the PC waveguide mode. The progressive adaptation requires the manufacture of holes with lateral dimensions smaller than those of the PC waveguide, see Fig. 3(a). These tiny holes effectively generate artificial dielectric layers with progressively varying effective indices. They are likely to be difficult to fabricate with deep etches and for this reason, in Fig. 3(b), large holes are assumed to be deeper than small holes. This assumption 


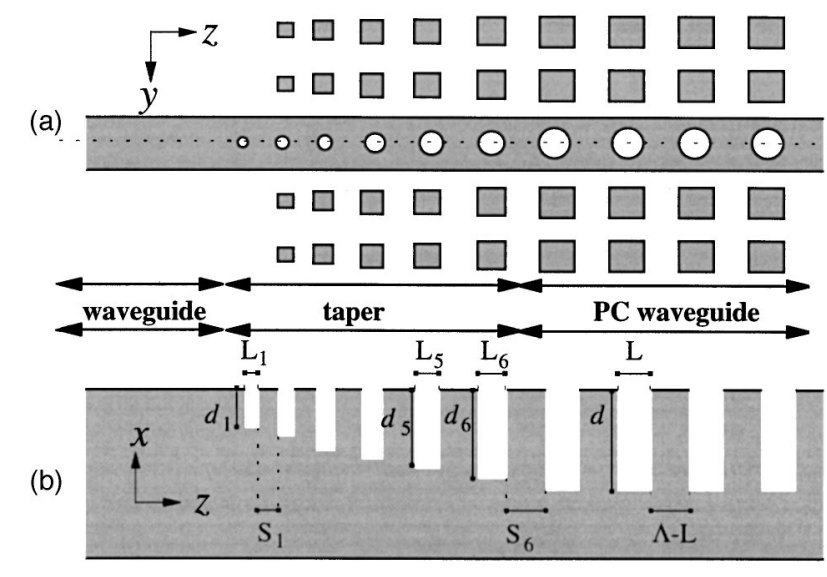

FIG. 3. Adiabatic taper for coupling into PC waveguides. Gray zones correspond to a high refractive-index material. (a) Top view. The PC waveguide shown for the sake of illustration is a ridge waveguide perforated by a 1D array of air holes and surrounded by a 2D PC composed of a square array of square pillars. (b) Cross section in the $x z$ plane shown in (a) with a dashed line. For fabrication constraints, large holes are assumed to be etched deeper than small ones.

is reasonable with respect to well-known properties of conventional etching processes.

To confirm the taper principle, let us consider the same SOI waveguide as in Fig. $2(L=80 \mathrm{~nm}, \Lambda=275 \mathrm{~nm}$, and $205 \mathrm{~nm}$ core thickness). Figure 4 shows a comparison between tapered and nontapered situations. The numerical results are all obtained with exact electromagnetic theory. ${ }^{16}$ The nontapered situation corresponds to the intrinsic case $(d$ $=500 \mathrm{~nm}$ ). The tapered situation holds for Bragg reflector with a groove depth of $160 \mathrm{~nm}$ (the core is partially etched). It is worth mentioning that, for $d=160 \mathrm{~nm}$, the loss exceeds $30 \%$ in the absence of taper for a guided wave with a frequency in the central part of the gap that impinges on a PC waveguide. In Fig. 4(a), the dashed curve shows the reflection spectrum of a ten-groove reflector with $d=500 \mathrm{~nm}$. The solid curve is obtained with a ten-groove reflector for $d$ $=160 \mathrm{~nm}$, but with a six-groove taper at the front face of the reflector. Figure 4(b) shows the transmission spectra of a SOI


FIG. 4. Illustration of the performance improvement achieved by tapering. The numerical results are obtained for the same SOI waveguide as in Fig. 2. Dashed curves are obtained without tapering for $d=500 \mathrm{~nm}$ (intrinsic case). Solid curves are obtained for $d=160$ with a taper formed by a series of 6 grooves with $L_{i}=20,30,40,50,60,70 \mathrm{~nm}$, with $d_{i}(\mathrm{~nm})=2.5\left(L_{i}-L_{1}\right)+$ 10 and with all the $S_{i}$ 's equal to $\Lambda-\mathrm{L}=195 \mathrm{~nm}$. (a) Reflection spectra computed for a ten-groove reflector. (b) Cavity transmission spectra computed near the peak resonance. Dashed curve: the cavity is formed by a $1.257 \mu \mathrm{m}$ long defect inserted between two five-groove Bragg reflectors. Solid curve: the cavity is formed by a $1.3 \mu \mathrm{m}$ long defect surrounded by two tapered mirrors composed of a taper, a three-groove reflector and a taper again. cavity around the resonance wavelength without tapering, dashed curve, and with tapering, solid curve. For the nontapered case, the cavity is formed by a $1.3 \mu \mathrm{m}$ long defect surrounded by two five-groove reflectors with $d=500 \mathrm{~nm}$. The tapered cavity is obtained by adding four identical sixgroove tapers at the four reflector extremities, while the reflector etch depth $d$ is only $160 \mathrm{~nm}$. Clearly, the tapered cavity with $160 \mathrm{~nm}$ deep slots (peak transmission above 98\% and $Q \approx 2600$ ) outperforms the intrinsic one with $500 \mathrm{~nm}$ deep slots. In our opinion, this excellent performance clearly indicates that very deep etches for intrinsic operation are not necessarily required for efficient coupling in many future applications.

In this letter, we have proposed a model for the out-ofplane losses encountered when light interacts with PC waveguides with low- or high-index confinements. The model makes clear that the loss comes from a mode mismatch and that the Bloch wave of the periodic structure (even if the PC waveguide is only a few periods long) is the key electromagnetic quantity of concern for studying the mismatch. Although the model was validated only for planar PC waveguides composed of 1D arrays of grooves, it seems reasonable that it applies as well for more complex structures since the model relies solely on the periodicity in the propagation direction. Adiabatic mode converters based on progressively adaptive geometries relying on narrow and tiny etches might be expected to play a significant role in photonic circuits based on PC waveguides.

${ }^{1}$ T. F. Krauss, R. M. De La Rue, and S. Brand, Nature (London) 383, 699 (1996).

${ }^{2}$ J. S. Foresi, P. R. Villeneuve, J. Ferrera, E. R. Thoen, G. Steinmeyer, S. Fan, J. D. Joannopoulos, L. C. Kimerling, H. I. Smith, and E. P. Ippen, Nature (London) 390, 143 (1997).

${ }^{3}$ Y. Yuan, T. Brock, P. Bhattacharya, C. Caneau, and R. Bhat, IEEE Photonics Technol. Lett. 9, 881 (1997).

${ }^{4}$ T. F. Krauss, O. Painter, A. Scherer, J. S. Roberts, and R. M. De La Rue, Opt. Eng. 37, 1143 (1998).

${ }^{5}$ A. Mekis, J. C. Chen, I. Kurland, S. Fan, P. R. Villeneuve, and J. D. Joannopoulos, Phys. Rev. Lett. 77, 3787 (1996).

${ }^{6}$ M. Tokushima, H. Kosaka, A. Tomita, and H. Yamada, Appl. Phys. Lett. 76, 952 (2000).

${ }^{7}$ This amounts to assume that the PC waveguide mode is below the vacuum line. Such situations are favored for strong index confinements, for firstorder PC waveguides, and for narrow etches (the effective index of the PC mode, that is smaller than that of the waveguide because of the removal of high-index material in the holes, has to remain larger than the refractive index of the substrate).

${ }^{8}$ H. Benisty, D. Labilloy, C. Weisbuch, C. J. M. Smith, T. F. Krauss, D. Cassagne, A. Béraud, and C. Jouanin, Appl. Phys. Lett. 76, 532 (2000).

${ }^{9}$ V. Berger, I. Pavel, E. Ducloux, and F. Lafon, J. Appl. Phys. 82, 5300 (1997).

${ }^{10}$ B. d'Urso, O. Painter, J. O'Brien, T. Tombrello, A. Yariv, and A. Scherer, J. Opt. Soc. Am. B 15, 1155 (1998).

${ }^{11}$ P. R. Villeneuve, S. Fan, S. G. Johnson, and J. D. Joannopoulos, IEE Proc.-Optoelectron. 6, 384 (1998).

${ }^{12} \mathrm{Ph}$. Lalanne and H. Benisty, J. Appl. Phys. 89, 1512 (2001).

${ }^{13}$ COST 268 project (see the website: www.ele.kth.se/COST268/WG2/ WG2.html for more information).

${ }^{14}$ D. Marcuse, Theory of Dielectric Optical Waveguides (Academic, New York, 1991).

${ }^{15}$ The Bloch waves are defined as the eigenvectors of the transfert matrix relating two planes $z=z_{1}$ and $z=z_{2}$ of the PC waveguide with $z_{2}-z_{1}=\Lambda$, see P. Yeh, Optical Waves in Layered Media (Wiley, New York, 1988).

${ }^{16}$ P. Lalanne and E. Silberstein, Opt. Lett. 25, 1092 (2000).

${ }^{17}$ This is obvious when considering that the etched and unpatterned membranes present a mirror symmetry with respect to the horizontal $y z$ plane in the center of the waveguide.

${ }^{18}$ Y. Xu, R. K. Lee, and A. Yariv, Opt. Lett. 25, 755 (2000). 